\title{
The Dynamical Instability of Nonrelativistic Many-Body Systems ${ }^{\star}$
}

\author{
Charles Radin \\ Department of Mathematics, University of Pennsylvania, Philadelphia, PA 19174, \\ and Department of Mathematics, University of Texas, Austin, TX 78712 ${ }^{\star \star}$, USA
}

\begin{abstract}
From computations in an exactly solvable many-body dynamical model we argue that, quite generally, a nonrelativistic quantum mechanics of infinitely many interacting particles must admit states without a global time evolution; equivalently, that the (quasi-local) observables of any such theory are not preserved in time by the Heisenberg dynamics. Our analysis, is based on a dynamical instability common to interacting finite-particle systems.
\end{abstract}

\section{Introduction}

The dynamics of many-body systems is known to be less singular in (nonrelativistic) quantum mechanics than in (nonrelativistic) classical mechanics. For example it remains a famous unsolved problem in classical mechanics [1] whether or not there is a global time evolution for each initial state in the $N$-body coulomb problem $(N \geqq 4$ point particles in three dimensions with attractive coulomb interaction) excluding the set, of measure zero [2], of those initial states which lead to collisions. Yet such difficulties are smoothed out in quantum mechanics by the wavefunction formalism. For any interaction given by a Kato potential, a class that includes the coulomb interaction [3], it has been proven [4] that every physical initial state has a global time evolution which remains physical in time. (By a "physical" state we understood one in which the usual particle observables, such as position, momentum and energy, are well defined.) Thus there can be no "catastrophe", or breakdown in the time evolution, of any initial state in a quantum mechanics of the most important dynamical models.

However the above argument does not preclude the phenomenon of "dynamical instability" for $N$-body quantum models, that is, a potential breakdown in the dynamics which gets more severe as larger $N$ are considered, which is manifested by an actual breakdown in the dynamics of the associated infiniteparticle models (and is thus qualitatively as significant as a breakdown for large enough $N$ ).

* Work supported in part by the National Science Foundation

$\star \star$ Present address 
For an example of such a dynamical instability in classical mechanics consider the model of $N \gg 1$ equal mass, point particles in one space dimension, with the $j^{\text {th }}$ particle at position $x_{j}(0)=j$ and with velocity $k_{j}(0)=-j^{2}$ at time $t=0$. Assume that the particles only interact through a hard core so that when two particles collide they exchange velocities but retain their order on the line. Then at any time $t>1 / N$ the first particle has velocity $k_{1}(t)=-N^{2}$. Applying this analysis to the appropriate system of infinitely many particles, it is clear that the analogous initial state (represented by the ordered set $\left\{\left(x_{j}(0), k_{j}(0)\right) \mid j=1,2, \ldots\right\}$ of infinitely many pairs of coordinates) cannot have a well defined time evolution $\left\{\left(x_{j}(t)\right.\right.$, $\left.\left.k_{j}(t)\right) \mid j=1,2, \ldots\right\}$ for (any) future time $t>0$.

This phenomenon (viewed equivalently as a dynamical instability of the $N$-particle model or as a catastrophe in the infinite-particle model-see [5] for details on this matter) can be controlled to some extent in classical mechanics (though obviously not yet for the coulomb interaction!) by isolating the troublesome initial states, showing them to be a set of measure zero for relevant measures [6-8]. Since quantum mechanics smooths out the dynamics of $N$-body models as described above, the question arises whether or not this phenomenon of dynamical instability of $N$-body models is also removed by the quantum formalism.

In Section 2 we will mimic the above classical mechanical instability for the analogous quantum mechanical model, and then argue in Section 3 that in any nonrelativistic quantum model with sufficiently strong repulsive core in the interaction (and at least two species of particles if identical particles are used) the instability will remain. We then reformulate this result to show that in any (quasi-) local observable theory of infinite-particle nonrelativistic dynamics, the set of observables cannot be preserved in time if the interaction is sufficiently repulsive. Finally we discuss briefly in what manner this requires a re-evaluation of the $C^{*}$-algebraic nonrelativistic dynamical theories in [9] and its progeny.

\section{An Exactly Solvable Dynamical Model}

We consider in this section the dynamical model governed by the Schrödinger equation for a finite number, $N \geqq 2$, of distinguishable, equal mass, point particles in one space dimension, whose only interaction is a hard core; that is, the particles are assumed impenetrable to one another but otherwise free. A wavefunction for our system is thus a normalized, Lebesgue-square-integrable function on $\mathbb{R}^{N}$, and our Hamiltonian is $-\frac{1}{2} \sum_{j=1}^{N}\left(\partial^{2} / \partial x_{j}^{2}\right)$ with Dirichlet boundary conditions (see [11], esp. VI- $\$ 1.3$ and VI- $\S 4.4)$ on the set $T=\left\{\boldsymbol{x}=\left(x_{1}, \ldots, x_{N}\right) \in \mathbb{R}^{N} \mid x_{i}=x_{j}\right.$ for some $i \neq j\}$.

Let $S_{N}$ be the set of permutations $P$ on the first $N$ natural numbers, with action denoted $P: j \rightarrow P(j)$, let $D=\left\{\boldsymbol{x} \in \mathbb{R}^{N} \mid x_{1}<x_{2}<\ldots<x_{N}\right\}$ and let $\chi$ be the characteristic function for $D$. We define the time dependent wavefunction $\Psi_{t}$ by

$$
\begin{aligned}
\Psi_{t}(\boldsymbol{x})= & \frac{\chi(\boldsymbol{x})}{C_{N}} \sum_{P \in S_{N}}(-1)^{|P|+1} \prod_{j=1}^{N} \frac{1}{(2 \pi)^{1 / 4} \pi^{1 / 2}} \\
& \cdot \int_{-\infty}^{\infty} \exp \left[i k_{j}\left(x_{P(j)}-a_{j}-k_{j} t / 2\right)-\left(k_{j}-b_{j}\right)^{2}\right] d k_{j},
\end{aligned}
$$


where $|P|= \pm 1$ is the parity of $P$, where $a_{j}$ and $b_{j}$ are fixed throughout this paper to have the values

$$
b_{j}=-a_{j}^{2} ; \quad a_{j}=\exp [100(j+1) !],
$$

and $C_{N}$ is the positive normalization constant (independent of $\boldsymbol{x}$ and $t$ and with uniform lower bound determined below) such that the $L_{2}$-norm $\left\|\Psi_{t}(\cdot)\right\|=1$ for all $t$. It is easily seen that for all $t, \Psi_{t}$ is a continuous function of $\boldsymbol{x}$, which vanishes for $\boldsymbol{x}$ in $T$, and is such that

$$
\frac{\partial}{\partial t} \Psi_{t}(\boldsymbol{x})=-\frac{1}{2 i} \sum_{j=1}^{N} \frac{\partial^{2}}{\partial x_{j}^{2}} \Psi_{t}(\boldsymbol{x}), \text { for all }(\boldsymbol{x}, t) \text { in } T^{c} \times \mathbb{R},
$$

where $T^{\mathrm{t}}$ is the complement of $T$ in $\mathbb{R}^{N}$. (We note that the form of our $\Psi_{t}$ was inspired by calculations in [10].) Using this it is easy to show that

$$
\Psi_{t}=\frac{\chi}{C_{N}} \sum_{P \in S_{N}}(-1)^{|P|+1} \psi_{P, t},
$$

where for all $\boldsymbol{x}$ in $\mathbb{R}^{N}$ and $t$ in $\mathbb{R}$ we define

$$
\begin{aligned}
\Psi_{P, t}(\boldsymbol{x})=\prod_{j=1}^{N} \frac{1}{(2 \pi)^{1 / 4}(1+i t / 2)^{1 / 2}} & \exp \left[i\left(b_{j} x_{P(j)}-a_{j} b_{j}-b_{j}^{2} t / 2\right)\right. \\
& \left.-\left(x_{P(j)}-a_{j}-b_{j} t\right)^{2} /(4+2 i t)\right] .
\end{aligned}
$$

We note that $\left\|\Psi_{P, t}(\cdot)\right\|=1$ for all $t$ and $P$.

The immediate goal of this section is to show that the speed of the particle labeled by $j=1$ undergoes a large increase as time passes $t=0$, an increase which in fact diverges as a function of the total particle number $N$, in agreement with the classical analog of Section 1. As a necessary step in the argument it is shown that at times $t \cong 0$ and $t \cong a_{N}^{-1}$ the sum of all contributions to $\Psi_{t}$ of the various $\psi_{P, t}$ in (1) are negligable except for one (different for the two times) and that therefore the system can be treated as a sequence of well localized and isolated particles at these times.

We begin with some notation. We will need two decompositions of $S_{N}$; using the notation $I$ for the identity permutation and $M$ for the permutation defined by:

$$
M(j)= \begin{cases}j+1, & j \leqq N-1 \\ 1, & j=N\end{cases}
$$

we define

$$
R_{k}=\left\{\begin{array}{l}
\{I\}, \quad k=1 \\
\left\{P \in S_{N} \mid k \text { is the largest value of } j \text { such that } P(j) \neq j\right\}, \quad k \geqq 2
\end{array}\right.
$$

and

$$
T_{k}=\left\{\begin{array}{l}
\{M\}, \quad \text { if } \quad k=1 \\
\left\{P \in S_{N} \mid k \text { is the largest value of } j \text { such that } P(j) \neq M(j)\right\}, \quad \text { if } k \geqq 2 .
\end{array}\right.
$$


Note that the cardinality of $R_{k}$ and of $T_{k}$ is $(k-1)(k-1)$ ! for $k \geqq 2$. Finally we define $\tilde{a}_{j}=a_{j}\left(1-a_{j} / a_{N}\right)$ and $\bar{a}_{j}=2 a_{j}^{2} /\left(1+4 a_{j}^{2}\right)$.

We will also need the following two simple estimates. If $K \geqq 0$ and $d>0$, then

$$
\begin{aligned}
\int_{K}^{\infty} \exp \left(-x^{2} / d\right) d x & =\int_{0}^{\infty} \exp \left[-(y+K)^{2} / d\right] d y \\
& \leqq \int_{0}^{\infty} \exp \left[-\left(y^{2}+K^{2}\right) / d\right] d y \\
& \leqq \exp \left(-K^{2} / d\right) \int_{0}^{\infty} \exp \left(-y^{2} / d\right) d y \\
& \leqq\left[(\pi d)^{1 / 2} / 2\right] \exp \left(-K^{2} / d\right) .
\end{aligned}
$$

Next assume $a \geqq 0, d>0$, and define

$$
\begin{aligned}
J_{1}= & \int_{x>3 a / 2} x^{2} \exp \left[-(x-a)^{2} / d\right] d x \\
= & \int_{0}^{\infty}(y+3 a / 2)^{2} \exp \left[-(y+a / 2)^{2} / d\right] d y \\
\leqq & \exp \left[-a^{2} / 4 d\right] \int_{0}^{\infty}(y+3 a / 2)^{2} \exp \left(-y^{2} / d\right) d y \\
\leqq & \exp \left[-a^{2} / 4 d\right]\left[\int_{0}^{\infty} y^{2} \exp \left(-y^{2} / d\right) d y\right. \\
& \left.+3 a \int_{0}^{\infty} y \exp \left(-y^{2} / d\right) d y+\left(9 a^{2} / 4\right) \int_{0}^{\infty} \exp \left(-y^{2} / d\right) d y\right] \\
\leqq & \exp \left[-a^{2} / 4 d\right]\left[(\pi d)^{1 / 2} d / 4+3 a d / 2+(\pi d)^{1 / 2} 9 a^{2} / 8\right] .
\end{aligned}
$$

Then define

$$
\begin{aligned}
J_{2}= & \int_{x<a / 2} x^{2} \exp \left[-(x-a)^{2} / d\right] d y \\
= & \int_{-\infty}^{0}(y+a / 2)^{2} \exp \left[-(y-a / 2)^{2} / d\right] d y \\
= & \int_{0}^{\infty}(z-a / 2)^{2} \exp \left[-(z+a / 2)^{2} d\right] d z \\
\leqq & \exp \left[-a^{2} / 4 d\right] \int_{0}^{\infty}(z-a / 2)^{2} \exp \left(-z^{2} / d\right) d z \\
\leqq & \exp \left[-a^{2} / 4 d\right]\left[\int_{0}^{\infty} z^{2} \exp \left(-z^{2} / d\right) d z\right. \\
& \left.-a \int_{0}^{\infty} z \exp \left(-z^{2} / d\right) d z+\left(a^{2} / 4\right) \int_{0}^{\infty} \exp \left(-z^{2} / d\right) d z\right] \\
\leqq & \exp \left[-a^{2} / 4 d\right]\left[(\pi d)^{1 / 2} d / 4-a d / 2+(\pi d)^{1 / 2} a^{2} / 8\right]
\end{aligned}
$$


Finally we define

$$
\begin{aligned}
J=J_{1}+J_{2} & =\int_{|x-a|>a / 2} x^{2} \exp \left[-(x-a)^{2} / d\right] d x \\
& \leqq \exp \left[-a^{2} / 4 d\right]\left[(\pi d)^{1 / 2}\left(d / 2+5 a^{2} / 4\right)+a d\right] .
\end{aligned}
$$

We now begin our specific estimates of the various $\left\|\chi \psi_{P, t}\right\|$ and related quantities.

Assume $P \in T_{k}, k \neq 1$. Setting $y=x_{P(k-1)}, z=x_{k}$, and $h=P^{-1}(k)$,

$$
\begin{aligned}
\left\|\chi \psi_{P, a_{N}-1}\right\|= & \int_{D}\left(\bar{a}_{N} / \pi\right)^{N / 2} \prod_{j=1}^{N} \exp \left[-\bar{a}_{N}\left(x_{P(j)}-\tilde{a}_{j}\right)^{2}\right] d x \\
\leqq & \left(\bar{a}_{N} / \pi\right) \int_{z>y} \exp \left[-a_{N}\left(z-\tilde{a}_{h}\right)^{2}-\bar{a}_{N}\left(y-\tilde{a}_{k-1}\right)^{2}\right] d y d z \\
\leqq & \left.\left(\bar{a}_{N} / \pi\right) \int_{\left|y-\tilde{a}_{k-1}\right|>\tilde{a}_{k-1} / 2} \exp \left[-\bar{a}_{N}\left(y-\tilde{a}_{k-1}\right)^{2}\right] d y\right] \\
& \cdot\left[\int_{\left|z-\tilde{a}_{h}\right|>\tilde{a}_{h} / 2} \exp \left[-\bar{a}_{N}\left(z-\tilde{a}_{h}\right)^{2}\right] d z\right] \\
\leqq & \left(\bar{a}_{N} / \pi\right)\left(\pi / \bar{a}_{N}\right)^{1 / 2}\left(\pi / \bar{a}_{N}\right)^{1 / 2} \exp \left[-\left(\bar{a}_{N} / 4\right)\left(\tilde{a}_{k-1}^{2}+\tilde{a}_{h}^{2}\right)\right] \\
\leqq & \exp \left(-\tilde{a}_{k-1}^{2} / 10\right) \leqq \exp \left(-a_{k-1}^{2} / 40\right), \quad \text { and so } \\
\left\|\chi \psi_{P, a_{N}-1}\right\| \leqq & \exp \left(-a_{k-1}^{2} / 80\right) .
\end{aligned}
$$

Next we note that (treating $j=N$ specially)

$$
\begin{aligned}
\left\|\chi \psi_{M, a_{N}-1}\right\|^{2}= & \int_{D}\left(\bar{a}_{N} / \pi\right)^{N / 2} \prod_{j=1}^{N} \exp \left[-\bar{a}_{N}\left(x_{M(j)}-\tilde{a}_{j}\right)^{2}\right] d x \\
\geqq & \left(\bar{a}_{N} / \pi\right)^{N / 2}\left(\prod_{j=1}^{N-1} \int_{a_{j} / 2}^{a_{j} / 2} \exp \left(-\bar{a}_{N} x^{2}\right) d x\right) \\
& \cdot \int_{-a_{1} / 4}^{a_{1} / 4} \exp \left(-a_{N} x^{2}\right) d x \\
\geqq & \left(\bar{a}_{N} / \pi\right)^{N / 2}\left(\prod_{j=1}^{N-1}\left(\pi / \bar{a}_{N}\right)^{1 / 2}\left(1-\exp \left[-\bar{a}_{N} \tilde{a}_{j}^{2} / 4\right]\right)\right) \\
& \cdot\left(1-\exp \left[-\bar{a}_{N} \tilde{a}_{1} / 9\right]\right) \\
\geqq & \left(\prod_{j=1}^{N-1}\left[1-\exp \left(-a_{j}^{2} / 40\right)\right]\right)\left(1-\exp \left[-a_{1}^{2} / 80\right]\right) \\
\geqq & \left(\exp \sum_{j=1}^{N-1} \ln \left[1-\exp \left(-a_{j}^{2} / 40\right)\right]\right)\left(1-\exp \left[-a_{1}^{2} / 80\right]\right) \\
\geqq & {\left[\exp \sum_{j=1}^{N-1}-(3 / 2) \exp \left(-a_{j}^{2} / 40\right)\right]\left[1-\exp \left(-a_{1}^{2} / 80\right)\right], }
\end{aligned}
$$

where we have used the inequality $\ln (1+y) \geqq 3 y / 2$, valid for $-\frac{1}{2}<y \leqq 0$. Therefore we have

$\left\|\chi \psi_{M, a_{N}^{-1}}\right\| \geqq\left[\exp -\sum_{j=1}^{N-1} \exp \left(-a_{j}^{2} / 40\right)\right]\left[1-\exp \left(-a_{1}^{2} / 80\right)\right]$. 
Next we need an estimate for the inner product

$$
\begin{aligned}
\left\langle\bar{\Psi}_{a_{N^{-1}}}, x_{1} \bar{\Psi}_{a_{N^{-1}}}\right\rangle & =C_{N}^{-1} \sum_{Q \in S_{N}}(-1)^{|P|+1}\left\langle\bar{\Psi}_{\left.a_{N^{-1}}, x_{1} \chi \psi_{P, a_{N}^{-1}}\right\rangle}\right. \\
& \leqq C_{N}^{-1} \sum_{Q \in S_{N}}\left\|x_{1} \chi \psi_{P, a_{N^{-1}}}\right\| .
\end{aligned}
$$

Assume $Q \in T_{k}, k \neq 1$ and also that $Q^{-1}(1) \neq k-1$. Then if we let $h=Q^{-1}(k)$, $y=x_{Q(k-1)}, z=x_{k}$ and $f=Q^{-1}(1)$, we have

$$
\begin{aligned}
\left\|x_{1} \chi \psi_{Q, a_{N^{-1}}}\right\|^{2}= & \int_{D}\left(\bar{a}_{N} / \pi\right)^{N / 2} x_{1}^{2} \prod_{j=1}^{N} \exp \left[-\bar{a}_{N}\left(x_{Q(j)}-\tilde{a}_{j}\right)^{2}\right] d x \\
\leqq & \left(\bar{a}_{N} / \pi\right)^{3 / 2} \int_{z>y} \exp \left[-\bar{a}_{N}\left(z-\tilde{a}_{h}\right)-\bar{a}_{N}\left(y-\tilde{a}_{k-1}\right)\right] d y d z \\
& \cdot \int_{-\infty}^{\infty} x_{1}^{2} \exp \left[-\bar{a}_{N}\left(x_{1}-\tilde{a}_{f}\right)^{2} d x_{1}\right. \\
\leqq & \exp \left(-a_{k-1}^{2} / 40\right)\left(\bar{a}_{N} / \pi\right)^{1 / 2} \int_{-\infty}^{\infty} x_{1}^{2} \exp \left[-\bar{a}_{N}\left(x_{1}-\tilde{a}_{f}\right)^{2}\right] d x_{1} \\
\leqq & \exp \left(-a_{k-1}^{2} / 40\right)\left[\tilde{a}_{f}^{2}+\left(2 \bar{a}_{N}\right)^{-1}\right] \\
\leqq & \exp \left(-a_{k-1}^{2} / 40\right)\left[a_{k-1}^{2}+2\right] .
\end{aligned}
$$

If however $Q \in T_{k}, k \neq 1$ and also $Q^{-1}(1)=k-1$, then in a similar vein but now using (3), we have

$$
\begin{aligned}
\left\|x_{1} \chi \psi_{Q, a_{N}-1}\right\|^{2} \leqq & \left(\bar{a}_{N} / \pi\right) \int_{z>y} y^{2} \exp \left[-\bar{a}_{N}\left(z-\tilde{a}_{h}\right)^{2}-\bar{a}_{N}\left(y-\tilde{a}_{k-1}\right)^{2}\right] d y d z \\
\leqq & \left(\bar{a}_{N} / \pi\right) \int_{\left|y-\tilde{a}_{k-1}\right|>\tilde{a}_{k-1 / 2}} y^{2} \exp \left[-\bar{a}_{N}\left(y-\tilde{a}_{k-1}\right)^{2}\right] d y \\
& \cdot \int_{\left|z-\tilde{a}_{h}\right|>\tilde{a}_{h} / 2} \exp \left[-\bar{a}_{N}\left(z-a_{h}\right) 2\right] d z \\
\leqq & 2 \tilde{a}_{k-1}^{2} \exp \left(-a_{k-1}^{2} / 40\right) \\
\leqq & 2 a_{k-1}^{2} \exp \left(-a_{k-1}^{2} / 40\right)
\end{aligned}
$$

So in general if $Q \in T_{k}, k \neq 1$ then

$$
\left\|x_{1} \chi \psi_{Q, a_{N^{-1}}}\right\| \leqq 2 a_{k-1} \exp \left(-a_{k-1}^{2} / 80\right) .
$$

Finally, taking $Q=M$, we have

$$
\begin{aligned}
\left\|x_{1} \chi \psi_{M, a_{N}-1}\right\|^{2} & \leqq\left(\bar{a}_{N} / \pi\right)^{1 / 2} \int_{-\infty}^{\infty} x^{2} \exp \left(-\bar{a}_{N} x^{2}\right) d x \\
& \leqq\left(2 \bar{a}_{N}\right)^{-1} \leqq 2
\end{aligned}
$$

Therefore we have from (6), using (7) and (8),

$$
\left\langle\bar{\Psi}_{a_{N^{-1}}}, x_{1} \bar{\Psi}_{a_{N^{-1}}}\right\rangle \leqq C_{N}^{-1}\left(2+\sum_{k=2}^{N} k ! 2 a_{k-1} \exp \left(-a_{k-1}^{2} / 80\right)\right) .
$$


Inequality (9) is the basic overestimate that we need, pertaining to $t=a_{N}^{-1}$; we now need an underestimate corresponding to $t=0$. We will use

$$
\begin{aligned}
\left\langle\Psi_{0}, x_{1} \Psi_{0}\right\rangle \geqq & C_{N}^{-1}\left(\left\langle\Psi_{0}, x_{1} \chi \psi_{I, 0}\right\rangle-\sum_{P \neq I}\left\|x_{1} \chi \psi_{P, 0}\right\|\right) \\
\geqq & C_{N}^{-1}\left[C_{N}^{-1}\left(\left\langle\chi \psi_{I, 0}, x_{1} \chi \psi_{I, 0}\right\rangle-\sum_{P \neq I}\left\|x_{1} \chi \psi_{P, 0}\right\|\right)\right. \\
& \left.-\sum_{P \neq I}\left\|x_{1} \chi \psi_{P, 0}\right\|\right] .
\end{aligned}
$$

We begin with an underestimate for

$$
\begin{aligned}
\left\langle\chi \psi_{I, 0}, x_{1} \chi \psi_{I, 0}\right\rangle & =(2 \pi)^{-N / 2} \int_{D} x_{1} \prod_{j=1}^{N} \exp \left[-\left(x_{j}-a_{j}\right)^{2} / 2\right] d \boldsymbol{x} \\
& \geqq(2 \pi)^{-N / 2} \int_{D} x_{1} \prod_{j=1}^{N} \exp \left[-\left(x_{j}-a_{j}\right)^{2} / 2\right] d \boldsymbol{x},
\end{aligned}
$$

where $H=H_{1} \cup H_{2}$ and $H_{1}=\left\{\boldsymbol{x} \mid x_{1}<0\right\}, H_{2}=\left\{\boldsymbol{x}|| x_{j}-a_{j} \mid<a_{j} / 2, \forall j\right\}$.

Now

$$
\begin{aligned}
& (2 \pi)^{-N / 2} \int_{H_{1}} x_{1} \prod_{j=1}^{N} \exp \left[-\left(x_{j}-a_{j}\right)^{2} / 2\right] d x \\
& =(2 \pi)^{-1 / 2} \int_{-\infty}^{0} x \exp \left[-\left(x-a_{1}\right)^{2} / 2\right] d x \\
& \geqq-(2 \pi)^{-1 / 2} \int_{a_{1}}^{\infty}\left(y-a_{1}\right) \exp \left(-y^{2} / 2\right) d y \\
& \geqq-\exp \left(-a_{1}^{2} / 2\right) .
\end{aligned}
$$

And using (2) we find that

$$
\begin{aligned}
(2 \pi)^{-N / 2} & \left.\int_{H_{2}} x_{1} \prod_{j=1}^{N} \exp \left[-x_{j}-a_{j}\right)^{2} / 2\right] d x \\
= & (2 \pi)^{-N / 2}\left[\prod_{j=2}^{N} \int_{\left|x_{j}-a_{j}\right|<a_{j} / 2} \exp \left[-\left(x_{j}-a_{j}\right)^{2} / 2\right] d x_{j}\right] \\
& \cdot \int_{\left|x-a_{1}\right|<a_{1} / 2} x \exp \left[-\left(x-a_{1}\right)^{2} / 2\right] d x \\
\geqq & (2 \pi)^{-N / 2}\left[(2 \pi)^{(N-1) / 2} \prod_{j=2}^{N}\left(1-\exp \left[-a_{j}^{2} / 8\right]\right)\right] \\
& \cdot\left[a_{1}(2 \pi)^{1 / 2}\left(1-\exp \left[-a_{1}^{2} / 8\right]\right)\right] \\
\geqq & a_{1} \prod_{j=1}^{N}\left(1-\exp \left[-a_{j}^{2} / 8\right]\right) .
\end{aligned}
$$

Therefore from (11), using (12) and (13), we have

$$
\begin{aligned}
\left\langle\chi \psi_{I, 0}, x_{1} \chi \psi_{I, 0}\right\rangle & \geqq a_{1} \prod_{j=1}^{N}\left(1-\exp \left[-a_{j}^{2} / 8\right]\right)-\exp \left(-a_{1}^{2} / 2\right) \\
& \geqq a_{1} \exp \left(\sum_{j=1}^{N}-(3 / 2) \exp \left[-a_{j}^{2} / 8\right]\right)-\exp \left(-a_{1}^{2} / 2\right) .
\end{aligned}
$$


Next, assuming $Q \in R_{k}, k \neq 1$, we need an overestimate for $\left\|x_{1} \chi \psi_{Q, 0}\right\|$. Assume $Q^{-1}(1) \neq k$ and let $h=Q^{-1}(k), y=x_{Q(k)}, z=x_{k}$, and $f=Q^{-1}(1)$. Then

$$
\begin{aligned}
\left\|x_{1} \chi \psi_{Q, 0}\right\|^{2}= & \int_{D} x_{1}^{2}(2 \pi)^{-N / 2} \prod_{j=1}^{N} \exp \left[-\left(x_{Q(j)}-a_{j}\right)^{2} / 2\right] d x \\
\leqq & (2 \pi)^{-3 / 2} \int_{z>y} \exp \left[-\left(z-a_{h}\right)^{2} / 2-\left(y-a_{k}\right)^{2} / 2\right] d y d z \\
& \cdot \int_{-\infty}^{\infty} x_{1}^{2} \exp \left[-\left(x_{1}-a_{f}\right)^{2} / 2\right] d x_{1} \\
\leqq & (2 \pi)^{-3 / 2}\left[\int_{\left|y-a_{k}\right|>a_{k} / 2} \exp \left[-\left(y-a_{k}\right)^{2} / 2\right] d y\right] \\
& \cdot\left[\int_{\left|z-a_{h}\right|>a_{h} / 2} \exp \left[-\left(z-a_{h}\right)^{2} / 2\right] d z\right](2 \pi)^{1 / 2}\left(1+a_{f}^{2}\right) \\
\leqq & \left(1+a_{f}^{2}\right) \exp \left(-a_{k}^{2} / 8\right) \\
\leqq & 2 a_{k}^{2} \exp \left(-a_{k}^{2} / 8\right) .
\end{aligned}
$$

If however $Q \in R_{k}, k \neq 1$ and $Q^{-1}(1)=k$, then

$$
\begin{aligned}
\left\|x_{1} \chi \psi_{Q, 0}\right\|^{2} \leqq & (2 \pi)^{-1} \int_{z>y} y^{2} \exp \left[-\left(y-a_{k}\right)^{2} / 2-\left(z-a_{h}\right)^{2} / 2\right] d y d z \\
\leqq & (2 \pi)^{-1}\left[\int_{\left|y-a_{k}\right|>a_{k} / 2} y^{2} \exp \left[-\left(y-a_{k}\right)^{2} / 2\right] d y\right] \\
& \cdot\left[\int_{\left|z-a_{h}\right|>a_{h} / 2} \exp \left[-\left(z-a_{h}\right)^{2} / 2\right] d z\right] \\
\leqq & (2 \pi)^{-1}\left[(2 \pi)^{1 / 2} 2 a_{k}^{2} \exp \left(-a_{k}^{2} / 8\right)\right](2 \pi)^{1 / 2} \\
\leqq & 2 a_{k}^{2} \exp \left(-a_{k}^{2} / 8\right) .
\end{aligned}
$$

So in general if $Q \in R_{k}, k \neq 1$, then

$$
\left\|x_{1} \chi \psi_{Q, 0}\right\| \leqq 2 a_{k} \exp \left(-a_{k}^{2} / 16\right) \text {. }
$$

Finally we need to estimate $C_{N}$. By definition

$$
C_{N}=\left\|\sum_{P \in S_{N}}(-1)^{|P|+1} \chi \psi_{P, a_{N}^{-1}}\right\|
$$

so using (4) and (5) we have

$$
\begin{aligned}
& {\left[1-\exp \left(-a_{1}^{2} / 80\right)\right]\left[\exp -\sum_{j=1}^{N-1} \exp \left(-a_{j}^{2} / 40\right)\right]-\sum_{k=2}^{N} k ! \exp \left(-a_{k-1}^{2} / 80\right)} \\
& \quad \leqq C_{N} \leqq 1+\sum_{k=2}^{N} k ! \exp \left(-a_{k-1}^{2} / 80\right)
\end{aligned}
$$

Now using $a_{k}=\exp [100(k+1) !]$, we note that $k ! \exp \left(-a_{k-1}^{2} / 80\right)<\exp (-100 k)$, so

$$
\sum_{k=2}^{N} k ! \exp \left(-a_{k-1}^{2} / 80\right)<\sum_{k=1}^{N} \exp (-100 k)<\exp (-50) .
$$

Also, $a_{1}^{2} / 80>50$, so

$$
\begin{gathered}
{[1-\exp (-50)] \exp [-\exp (-50)]<C_{N}<1+\exp (-50), \text { so }} \\
\left|C_{N}-1\right|<\exp (-20)
\end{gathered}
$$


Also, $2 k ! a_{k-1} \exp \left(-a_{k-1}^{2} / 80\right)<\exp (-100 k)$, so

$$
\sum_{k=2}^{N} 2 k ! a_{k-1} \exp \left(-a_{k-1}^{2} / 80\right)<\exp (-50) \text {. }
$$

Therefore using (16) and (17) in (9) we have

$$
\begin{aligned}
\left\langle\Psi_{a_{N^{-1}}}, x_{1} \Psi_{a_{N^{-1}}}\right\rangle & \leqq(1-\exp [-20])^{-1}(2+\exp [-50]) \\
& <3 \text { for all } N .
\end{aligned}
$$

On the other hand

$$
\begin{gathered}
(3 / 2) \exp \left(-a_{j}^{2} / 8\right)<\exp (-100 j), \text { so } \\
(3 / 2) \sum_{j=1}^{N} \exp \left(-a_{j}^{2} / 8\right)<\exp (-50), \text { and thus from }(14) \text { we have } \\
\left\langle\chi \psi_{I, 0}, x_{1} \chi \psi_{P, 0}\right\rangle \geqq 100 \text {. Using }(15), \\
\sum_{P \neq I}\left\|x_{1} \chi \psi_{P, 0}\right\| \leqq \sum_{k=2}^{N} 2 k ! a_{k}^{2} \exp \left(-a_{k}^{2} / 8\right) \leqq \exp (-50),
\end{gathered}
$$

and so from (16) and (10),

$$
\begin{aligned}
\left\langle\Psi_{0}, x_{1} \Psi_{0}\right\rangle & \geqq[1+\exp (-20)]^{-1}[(1+\exp [-20])(100-\exp [-50])-\exp (-50)] \\
& \geqq 50 \text { for all } N .
\end{aligned}
$$

The main point of this section is not only the estimates (18) and (19) but also the demonstration that the quantum system behaves very much like the classical one, at times $t \cong 0$ and $t \cong a_{N}^{-1}$. With the above technique it is also straightforward but tedious to show that $\left\langle\Psi_{t}, x_{1} \Psi_{t}\right\rangle$ is Cauchy as $N \rightarrow \infty$, for $t=0$ and $t=a_{N}^{-1}$; we leave the details to the reader. This given, inequalities (18) and (19) show that, as $N \rightarrow \infty$, particle 1 acquires infinite velocity at time $t=0$.

\section{Consequences of the Model}

We conclude by induction from the above calculations that in any nonrelativistic quantum mechanics of infinitely many particles, with a sufficiently repulsive core in the interaction, there exist initial states without a well defined tome evolution; equivalently, the set of observables of such a model is not preserved by the Heisenberg dynamics. This conclusion requires a number of supporting arguments and qualifications, which follow.

i) First of all we consider here only what we will call "complete" local observable theories or models. In a classical mechanical theory, point particles can be strictly localized in space, and a local observable model containing an infinite number of such distinguishable interacting particles, labeled by $j=1,2, \ldots$, would be "complete" only if every assignment $\left\{\left(x_{j}, k_{j}\right) \mid j=1,2, \ldots\right\}$ of positions $x_{j}$ and velocities $k_{j}$ which is meaningful locally (i.e. for which there are only finitely many particles in each bounded region of space, each with finite momentum and energy) is a state of the infinite-particle system. Such a restriction on a theory seems necessary if it is to have the flexibility to approximate arbitrary physical states of all the $N$-particle systems whose modeling is its raison d'être. In a nonrelativistic 
quantum mechanics strict localizability is an inappropriate notion due to instantaneous wavefunction spreading. However one can easily produce wavefunctions which represent particles which are well localized and separated in space and well localized in momentum and energy. In order to retain the same flexibility described above, it is therefore natural to require of any local observable theory the analogous notion of completeness.

ii) Our basic conclusion (underlined at the beginning of this section) but applied only to the one dimensional model of Section 2, follows from i) by the next argument. From our calculations, any complete local observable model of infinitely many distinguishable particles, with the given interaction, must admit an initial state $\tilde{\Psi}_{0}$ which in the region $\left(-a_{N+1} / 2, a_{N+1} / 2\right)$ would have the same properties as our $\Psi_{0}$. Since to be relevant such a model must give results approximating closely that of the appropriate finite-particle systems, if the time evolved state $\tilde{\Psi}_{t}$ existed for all $t$, it would have to describe a particle acquiring infinite velocity at $t=0$, which is incompatible with the notion of velocity. Finally, if the Heisenberg dynamics preserved the set of observables, which set defines the set of states, the dynamics of every initial state, including $\tilde{\Psi}_{0}$, would have to be defined leading to the same contradiction.

iii) The induction from our model with point hard core repulsive interaction to any "sufficiently repulsive" interaction for distinguishable particles seems reasonable. For higher space dimensions one has to control the directions of the various momenta, but since we are working in a finite time interval it would seem that wavefunction spreading would again be innocuous.

iv) There is one physical phenomenon which can interfere with the generalization of our arguments. If all the particles in the initial state are indistinguishable it can no longer be argued that the description of the system must break down in time. For example consider again the classical model of the introduction but with a description which does not distinguish particles. A state of the distinguishableparticle system was given by an ordered set of pairs $\left(x_{j}, k_{j}\right)$; a state for identical particles would thus consist of an unordered set of such pairs. For such identical particles, not only does the description of the system not break down in time, but in fact it is identical to that of the corresponding noninteracting system (which clearly has no difficulty of the type being described)! This is the case because the new symmetry does not allow the concept of a transfer of momentum to a particular particle, which is our basic mechanism. However this difficulty only arises in (impractically restrictive) models consisting of one species of identical particles. For models containing at least two particle species, $A$ and $B$, our argument can be modified as follows. For such a model we use coordinate $x_{1}$ of $\Psi_{t}$ to represent a single particle of species $A$ and (after symmetrization or antisymmetrization if necessary) we use the remaining coordinates to represent particles of species $B$. Therefore any local observable model which allows a complete description of at least two particle species (i.e. which admits as an initial state anything which for each bounded region is meaningful as a system of an arbitrary but finite number, $N_{j}$, of particles of species $j$ ) cannot have a well defined dynamics for all initial states if it uses a sufficiently repulsive interaction.

v) Finally, there is conspicuously absent any calculation using a specific $C^{*}$-algebra of quasi-local observables. This is purposeful, to emphasize our 
contention that our argument is more convincing than are any arguments known to us for the physical appropriateness of any particular $C^{*}$-algebra of observables.

At this point we note the relation of our results to certain previous work. In [12] it is argued that the dynamics, of nonrelativistic quantum systems of infinitely many particles, is not always given by automorphisms in local observable theories. This is argued from calculations with a specific $C^{*}$ - algebra, describing noninteracting bosons. However since no physical phenomenon (such as the dynamical instability we demonstrate for interacting systems) is shown to be at the basis of this result, it is tempting to ascribe the result to more superficial features of the specific $C^{*}$-algebra used and therefore to try to avoid the result by changing the algebra (an attitude reinforced by the fact that the free evolution preserves the analogous algebra for fermions). We feel that our argument should discourage such temptation, at least for interacting systems.

In conclusion, we view our analysis as a refinement of the local observable approach to the study of the dynamics of many-body systems, not as an obstacle to this approach. Indeed, we have already argued [5] that useful and general dynamical models can be constructed with automorphic Heisenberg dynamics if only one replaces the physical space $\mathbb{R}^{3}$ by $\mathbb{Z}^{3}$ (thus putting a "natural" cutoff on single particle velocities). Alternatively an effective velocity cutoff can be enforced by using a Lorentz invariant formalism, although this is somewhat impractical in that it only substitutes the more intractable singularities of particle production. Perhaps the most satisfying solution would be a method of controlling the underlying phenomenon of instability, by generalizing the classical mechanical tools (as used for example in [6-8]) to isolate those troublesome initial states by a "measure zero" technology, as we have attempted in [13].

Acknowledgments. It is a pleasure to acknowledge useful discussions with Rick Lavine, Henry Primakoff, and Barry Simon.

\section{References}

1. Sperling, H.J.: Bibliography on the singularities of the equations of motion of celestial mechanics (2nd ed.), NASA-George C. Marchall Space Flight Center, IN-AERO-70-I, Feb. 26, 1970

2. Saari, D. G.: Trans. Amer. Math. Soc. 162, 267 (1971); 168, 251 (1972)

3. Kato, T.: Trans. Amer. Math. Soc. 70, 195 (1951)

4. Hunziker, W.: J. Math. Phys. 7, 300 (1966)

5. Radin, C.: Commun. math. Phys. 44, 165 (1975)

6. Lanford, O. E.: Commun. math. Phys. 9, 179 (1968)

7. Lanford, O.E.: Commun. math. Phys. 11, 257 (1969)

8. Marchioro, C., Pellegrinotti, A., Presutti, E.: Commun. math. Phys. 40, 175 (1975)

9. Haag, R., Hugenholtz, N. M., Winnink, M.: Commun. math. Phys. 5, 215 (1967)

10. Yang, C. N.: Phys. Rev. 168, 1920 (1968)

11. Kato, T.: Perturbation theory for linear operators. Berlin-Heidelberg-New York: Springer 1966

12. Dubin, D. A., Sewell, G. L.: J. Math. Phys. 11, 2990 (1970)

13. Radin, C.: Commun. math. Phys. 33, 283 (1973)

Communicated by G. Gallavotti and J. L. Lebowitz 
